\title{
Dual Modes of Endoplasmic Reticulum-to-Golgi Transport in Dendrites Revealed by Live-Cell Imaging
}

\author{
April C. Horton ${ }^{1}$ and Michael D. Ehlers ${ }^{1,2,3}$ \\ Departments of ${ }^{1}$ Neurobiology, ${ }^{2}$ Cell Biology, and ${ }^{3}$ Pharmacology and Cancer Biology, Duke University Medical Center, Durham, North Carolina 27710
}

\begin{abstract}
Organelles of the neuronal secretory pathway are critical for the addition of membrane that accompanies neuronal development, as well as for the proper localization of plasma membrane proteins necessary for polarity, synaptic transmission, and plasticity. Here, we demonstrate that two organizations of the secretory pathway exist in neurons: one requiring processing of membrane and lipids in the Golgi complex of the cell body and one in which endoplasmic reticulum (ER)-to-Golgi trafficking is localized to dendrites. Using timelapse imaging of green fluorescent protein-tagged cargo proteins and compartment markers, we show that organelles of the secretory pathway, including ER, ER exit sites, and Golgi, are present and engage in trafficking in neuronal dendrites. We find that ER-to-Golgi trafficking involves highly mobile vesicular carriers that traffic in both the anterograde and retrograde directions throughout the dendritic arbor. Dendritic Golgi outposts, which appear developmentally during the phase of process outgrowth, are involved in the trafficking of both integral membrane proteins and the secreted neuronal growth factor BDNF. This distributed dendritic Golgi represents an organization of the secretory pathway unique among mammalian cells.
\end{abstract}

Key words: secretory pathway; protein trafficking; dendrite; local protein synthesis; Golgi; ER exit sites

\section{Introduction}

The addition of plasma membrane that accompanies polarization and process outgrowth is a fundamental task of the developing neuron (Futerman and Banker, 1996; Lecuit and Pilot, 2003). Both the addition of membrane and the localization of membrane constituents are dependent on the secretory pathway, the series of intracellular organelles consisting of the endoplasmic reticulum (ER), ER-Golgi intermediate compartment, and cis-, medial-, and trans-Golgi, which are specialized for the synthesis, targeting, and delivery of new membrane lipids and proteins (Nelson and Yeaman, 2001; Stephens and Pepperkok, 2001; Storrie and Nilsson, 2002). Despite a likely central role in neuronal morphogenesis and membrane trafficking, very little is known about the distribution, function, and regulation of secretory organelles or secretory transport pathways in neurons.

In non-neuronal cells, the organelles of the secretory pathway have a highly conserved spatial organization (Hirschberg et al., 1998; Hong, 1998; Lippincott-Schwartz et al., 2000; Antonny and Schekman, 2001). The ER extends throughout the cell, as do specialized ER exit sites, where coat protein complex II-coated vesicles containing

\footnotetext{
Received Jan. 10, 2003; revised May 9, 2003; accepted May 9, 2003.

This work was supported by grants from the National Institutes of Health (NIH), the American Heart Association, the Alzheimer's Association, the McKnight Foundation, the Klingenstein Fund, the Muscular Dystrophy Association, the Spinal Cord Research Foundation, the National Alliance for Research on Schizophrenia and Depression, the Sloan Foundation, the North Carolina Biotechnology Center, and the Ellison Medical Foundation (M.D.E.) and fellowships from the NIH Medical Scientist Training Program and the Ruth K. Broad Foundation (A.C.H.). We thank Kai Simons for VSVG3-SP-YFP, Jean-Pierre Paccaud for Sec24-YFP, Bai Lu for BDNF-GFP, Wanjin Hong for Sec13 antibody, Eunjoon Kim for Shank antibody, and Douglas Lyles for VSVG antibody. We also thank Chi Zhang and Haiwei Zhang for culturing neurons and Tom Blanpied, Juliet Hernandez, and Derek Scott for helpful comments on this manuscript.

Correspondence should be addressed to Dr. Michael D. Ehlers, Department of Neurobiology, Duke University Medical Center, Box 3209, Durham, NC 27710. E-mail: ehlers@neuro.duke.edu.

Copyright $\odot 2003$ Society for Neuroscience $\quad 0270-6474 / 03 / 236188-12 \$ 15.00 / 0$
}

nascent cargo bud off en route to the Golgi complex (Kuehn and Schekman, 1997; Stephens et al., 2000; Antonny and Schekman, 2001; Baumann and Walz, 2001). On emerging from ER exit sites, secretory carriers traffic centripetally to a perinuclear Golgi apparatus, in which protein processing and sorting occurs. Subsequently, post-Golgi carriers deliver their contents to the plasma membrane (Presley et al., 1997; Hirschberg et al., 1998). Although well established in model eukaryotic cells, it is unclear whether this canonical organization applies to neurons, given their immense size and surface area and their unique morphology and signaling requirements.

Recent studies suggest the presence of local dendritic secretory capacity. For example, trans-Golgi network-derived vesicles undergo calcium-evoked dendritic exocytosis (Maletic-Savatic and Malinow, 1998), and newly inserted glycine receptors and glutamate receptors appear rapidly on the surface of dendrites (Passafaro et al., 2001; Rosenberg et al., 2001). Such receptors may derive from dendritic membrane compartments immunopositive for secretory proteins (Pierce et al., 2000, 2001). Moreover, isolated dendrites retain the capacity to incorporate sugars (Torre and Steward, 1996) and translate membrane protein-encoding mRNAs (Kacharmina et al., 2000), consistent with Golgi-like function. Yet, whether the entire ER-to-Golgi secretory pathway or just late-stage post-Golgi vesicles contribute to dendritic secretory trafficking remains poorly understood.

In this study, we have used live-cell confocal imaging experiments along with immunocytochemistry in cultured hippocampal neurons to define the secretory pathway in dendrites. We demonstrate that newly synthesized membrane proteins concentrate at punctate immobile ER exit sites present throughout the dendrite, from which they bud into highly mobile carriers. These carriers then traffic either long distances to fuse with somatic Golgi or traffic locally to fuse with numerous distributed den- 
dritic Golgi outposts. These dendritic Golgi outposts are used not only by integral membrane proteins, but also by the secreted neuronal growth factor BDNF. Furthermore, the appearance of dendritic Golgi is developmentally regulated during the period of polarization and process outgrowth. These results reveal a novel distributed organization of the secretory pathway unique among mammalian cells.

\section{Materials and Methods}

DNA constructs. cDNA-encoding vesicular stomatitis virus glycoprotein ts045 fused to yellow-fluorescent protein (VSVG-YFP) (Toomre et al., 2000) was a gift from Kai Simons (Max-Planck-Institute of Molecular Cell Biology and Genetics, Dresden, Germany). ER-cyan fluorescent protein (CFP) and galactosyltransferase (GalTase)-CFP were obtained from Clontech (Palo Alto, CA). Sec24-YFP (Stephens et al., 2000) was a gift from Jean-Pierre Paccaud (University of Geneva, Geneva, Switzerland). BDNF-GFP was a gift from Bai Lu (National Institutes of Health, Bethesda, MD).

Neuronal culture and transfections. Primary cultures of hippocampal neurons were obtained from rat embryos at embryonic day 18 . The entire hippocampus was isolated and dissociated with trypsin, and cells were plated at 60,000 cells $/ \mathrm{cm}^{2}$ in Neurobasal medium (Sigma, St. Louis, MO) supplemented with B27, glutamax I, 5\% bovine serum, and $1 \mu \mathrm{g} / \mathrm{ml}$ gentamicin. 5-Fluorouracil deoxyribonucleoside $(10 \mu \mathrm{M})$ was added 6-7 $\mathrm{d}$ after plating, and cells were fed twice weekly thereafter with Neurobasal medium prepared as above but without bovine serum. Hippocampal neurons were grown at $37^{\circ} \mathrm{C}$ in $5 \% \mathrm{CO}_{2}$ on coverslips coated with polyD-lysine (Sigma). Neurons were transfected with Lipofectamine 2000 (Invitrogen, Carlsbad, CA) according to manufacturer's recommendations, except that $1.5 \mu \mathrm{g}$ of each DNA in $25 \mu \mathrm{l}$ Opti-MEM and $1 \mu \mathrm{l}$ of Lipofectamine 2000 in $25 \mu$ l Opti-MEM were mixed and added to coverslips in 12-well plates.

Immunocytochemistry. Immunocytochemistry was performed on hippocampal neurons using monoclonal antibodies against GM130 (Transduction Laboratories, San Diego, CA) and VSVG (a gift from Douglas Lyles, Wake Forest University, Winston-Salem, NC), and polyclonal antibodies against Sec13 (a gift from Wanjin Hong, National University of Singapore, Singapore) and Shank, a marker of excitatory synapses concentrated in dendritic spines (a gift from Eunjoon Kim, Korea Advanced Institute of Science and Technology, Daejeon, Korea). For GM130 staining, coverslips were processed essentially as described previously (Scott et al., 2001). For VSVG surface labeling, coverslips were processed as for GM130 staining, except that cells were not permeabilized with Triton $\mathrm{X}-100$. For Sec13 staining, coverslips were processed as described previously (Hammond and Glick, 2000), with the following modifications. Briefly, coverslips were rinsed with PBS and fixed in ice-cold methanol for $2 \mathrm{~min}$. Cells were rehydrated in PBS, pH 7.4, plus $0.1 \% N$-octyl- $\beta$-Dglucopyranoside containing $100 \mu \mathrm{M}$ bis(sulfosuccinimidyl) suberate (Pierce Chemical, Rockford, IL), for $30 \mathrm{~min}$ at room temperature. Cells were then quenched in $0.1 \%$ ethylenediamine- $\mathrm{HCl}, \mathrm{pH} 7.5$, for $15 \mathrm{~min}$. Coverslips were blocked for $1 \mathrm{hr}$ at $37^{\circ} \mathrm{C}$ in a buffer of $1 \%$ fish gelatin (Sigma, St. Louis, MO), $1 \%$ powdered milk, and $1 \%$ goat serum in PBS. Cells were then stained in blocking buffer at $4^{\circ} \mathrm{C}$ overnight. Sec13 antibody was used at a concentration of $0.02 \mathrm{mg} / \mathrm{ml}$. Coverslips were rinsed several times with blocking buffer, and secondary staining was performed in blocking buffer for $1 \mathrm{hr}$ at room temperature. Cells were then rinsed and mounted. For Shank staining, cells were fixed in $4 \%$ paraformaldehyde with $4 \%$ sucrose, and then permeabilized in $100 \%$ ice-cold methanol before blocking and staining.

Live-cell imaging. Twenty-four to $48 \mathrm{hr}$ after transfection, coverslips were imaged in a sealed chamber (Dagan, Minneapolis, MN) filled with imaging buffer consisting of the following (in $\mathrm{mM}$ ): $120 \mathrm{NaCl}, 3 \mathrm{KCl}, 2$ $\mathrm{CaCl}_{2}, 2 \mathrm{MgCl}_{2}, 10$ glucose, and 10 HEPES, pH 7.35, and $250 \mathrm{mOsm}$ to match culture growth medium. Wide-field epifluorescence images were acquired on a Nikon (Tokyo, Japan) TE300 inverted microscope. Confocal images were obtained using a Yokogawa spinning disk confocal (Solamere Technology Group, Salt Lake City, UT), with excitation lines from a 2.5 W Kr-Ar laser (Spectraphysics, Mountain View, CA) selected and shuttered via an acousto-optical tunable filter (Neos Technologies, Melbourne, FL) and emission directed through a filter wheel (Sutter Instruments, Novato, CA) holding bandpass filters (Chroma Technology, Brattleboro, VT). Images were acquired using a $100 \times$ or $60 \times$ Plan Apochromat objective and analyzed using Metamorph (Universal Imaging, West Chester, PA) with a 12-bit cooled CCD camera (Hamamatsu Inc., Bridgewater, $\mathrm{NJ}$ ). All live imaging experiments were performed at $32^{\circ} \mathrm{C}$. The stage was heated to $32^{\circ} \mathrm{C}$ using an airstream incubator (Nevtek, Burnsville, VA).

Analysis of carrier trafficking. The locations of VSVG-GFP carriers were tracked through stacks of images by marking the point of maximum intensity within each spot. Coordinates were recorded using the point tracker feature in Metamorph. Trajectories were then plotted using Origin (Microcal Software, Northampton, MA) and overlaid onto the image of the corresponding neuron. Carrier lifetime was defined as the point at which a carrier first appeared to when it was no longer evident, whether because it left the image frame or it merged with another structure.

Analysis of carrier budding. Average fluorescence in a region of constant area was tracked for VSVG-GFP- and Sec24-YFP-labeled puncta. Fluorescence was normalized to the average intensity of a region of the same size in a background region of the same frame.

Analysis of Golgi development. Maximum point projections were created of stacks of confocal images through entire neurons. Regions labeled for GM130 were manually traced. Golgi size was defined as the area of these GM130-labeled regions. Neurons with dendritic Golgi were defined as those that had any GM130 detectable beyond the neuronal soma.

Colocalization of VSVG-YFP and GalTase-CFP. Fluorescence intensity was determined for points along a linear region, five pixels in width, drawn down the length of the dendrite. These intensity values were plotted versus dendritic length, such that peaks on the plot indicate points of fluorescence accumulation. Values were background-subtracted and normalized. The extent of correlation between pixel intensities of each of the two fluorophores, YFP and CFP, was then calculated as a linear regression.

Statistical analysis. Error bars represent SEM. Statistical comparisons were $t$ tests.

\section{Results}

\section{Monitoring ER-to-Golgi trafficking in dendrites}

To monitor trafficking optically through the early secretory pathway in neuronal dendrites, we used the temperature-sensitive mutant of the vesicular stomatitis virus glycoprotein (ts045), tagged with spectral variants of green fluorescent protein (VSVGGFP) as a representative and classically studied membrane cargo protein (Bergmann, 1989; Presley et al., 1997; Hirschberg et al., 1998; Toomre et al., 2000). VSVG-GFP is misfolded and retained in the ER when expressing cells are incubated at the nonpermissive temperature $\left(39.5^{\circ} \mathrm{C}\right)$. On shifting to the permissive temperature $\left(32^{\circ} \mathrm{C}\right)$, VSVG-GFP correctly folds, rapidly exits the ER, and traffics to the Golgi (Presley et al., 1997; LippincottSchwartz et al., 2000). This temperature-dependent trafficking allows for direct visualization of a synchronous wave of newly synthesized membrane proteins through the secretory pathway in live neurons.

When neurons expressing VSVG-GFP were incubated at $39.5^{\circ} \mathrm{C}$ to accumulate VSVG in the ER, VSVG-GFP distributed throughout the dendrites and soma (Fig. 1A). This distribution was identical to the widespread ER distribution of both endogenous and exogenous ER resident proteins, including BiP, calnexin, $\operatorname{Trap} \alpha$, and protein disulfide isomerase (data not shown). Moreover, no VSVG-GFP was detectable at the cell surface (data not shown), confirming that at $39.5^{\circ} \mathrm{C}$, VSVG was trapped in the neuronal ER. After $2 \mathrm{hr}$ at the permissive temperature of $32^{\circ} \mathrm{C}$, surface labeling using an antibody against the luminal epitope of VSVG (Lefrancois and Lyles, 1982) revealed surface expression 
both on the neuronal soma (Fig. $1 B$ ) and the dendrites (Fig. 1C). We observed no discernible effect of temperature switch on neuronal morphology, health, or survival (data not shown). Thus, as VSVG-GFP progressed from the earliest secretory compartment, the ER, to the plasma membrane, it served as a useful representative membrane cargo protein to study secretory pathway dynamics in living neurons.

In neurons imaged live immediately after release from the $39.5^{\circ} \mathrm{C}$ block, VSVGGFP had a lacy, diffuse distribution, labeling the ER throughout the dendrites (Fig. $1 D$, top). Within minutes after switching to $32^{\circ} \mathrm{C}$, VSVG-GFP accumulated into scattered, uniformly sized puncta (Fig. $1 \mathrm{D}$, middle). By $15 \mathrm{~min}$ after release from the ER, VSVG-GFP was present in many highly mobile tubulovesicular carriers present throughout the dendritic arbor (Fig. $1 D$, bottom). To track the movement of individual carriers, time-lapse experiments were performed and the resulting trajectories were overlaid on images of dendrites. Such analysis revealed that early post-ER carriers traffic in both anterograde and retrograde directions (Fig. $1 E$ ). Figure $1 F$ shows frames from a typical time-lapse experiment of VSVG-GFP in a hippocampal dendrite within the first 15 min after release from the ER. In this example, a carrier (Fig. $1 F$, arrowhead) transported VSVG-GFP along a curvilinear path toward the neuronal soma. In the final frame, the carrier merges with the stable, stationary structure in the proximal dendrite (Fig. $1 F$, arrow). The total fluorescence of the carrier remained constant, and it moved as a single structure without branching or dividing, suggesting that mobile carriers retain their identity during long-range transport. These data indicate that VSVG-GFP is transported from the ER in carriers that traffic bidirectionally within the dendrites. Such bidirectional traffic of pre-Golgi carriers is inconsistent with a single centralized Golgi destination, and it is in marked contrast to the highly directional centripetal transport of pre-Golgi carriers in nonneuronal cells (Hong, 1998; Klumperman, 2000; LippincottSchwartz et al., 2000).

\section{Functional ER exit sites in distal dendrites}

Before leaving the ER proper, cargo accumulates at ER exit sites (Hong, 1998; Klumperman, 2000; Lippincott-Schwartz et al., 2000). Such sites are characterized by the presence of COPII coat proteins that mediate vesicle budding (Kuehn and Schekman, 1997; Tang et al., 1997; Kirchhausen, 2000). The appearance of a punctate concentration of VSVG-GFP 5 min after removal of the $39.5^{\circ} \mathrm{C}$ block (Fig. $1 D$ ) is reminiscent of cargo concentrating at ER exit sites before budding off into carriers destined for the Golgi complex (Stephens et al., 2000). To determine whether ER exit sites are present in neuronal dendrites, we examined the distribution of both endogenous and exogenous COPII proteins. Immunocytochemical staining for the COPII protein Sec13 revealed numerous uniformly sized

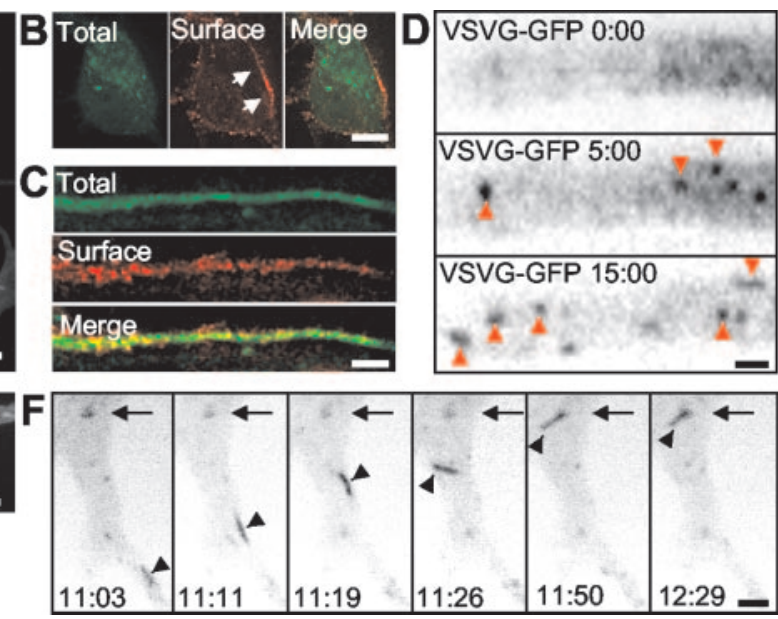

Figure 1. Secretory cargo exits the neuronal ER via vesiculotubular carriers that traffic bidirectionally in dendrites. $A$, the direction the neuronal soma. The carrier follows a curvilinear path until it reaches the persistent fluorescent structure at the top of the frame (arrow). The carrier then merges with this structure, presumably Golgi. Scale bar, $2.5 \mu \mathrm{m}$. See supplemental movie S3 (available at www.jneurosci.org) for time lapses.

puncta distributed throughout the dendrites (Fig. 2A, top). Sec13 colocalized with exogenously expressed Sec24-YFP (Fig. $2 \mathrm{~A}$, middle and bottom), another component of the COPII coat that labels ER exit sites in live cells (Stephens et al., 2000). Time-lapse imaging of Sec24-YFP indicated that dendritic ER exit sites are stably localized and seldom appear or disappear on the time scale of minutes (Fig. $2 B$ ).

To determine whether COPII-laden dendritic ER exit sites concentrate cargo, neurons expressing both VSVG-CFP and Sec24-YFP were imaged $5 \mathrm{~min}$ after release from $39.5^{\circ} \mathrm{C}$ block (Fig. 2C). At this time, VSVG-CFP accumulated at discrete spots throughout the dendrite labeled by Sec24-YFP (Fig. 2C), providing strong evidence that these puncta are bona fide dendritic ER exit sites. The functionality of these dendritic ER exit sites was confirmed in two-color confocal time-lapse imaging experiments of neurons coexpressing VSVG-CFP and Sec24-YFP. Within $5 \mathrm{~min}$ after the release from the $39.5^{\circ} \mathrm{C}$ block, VSVG-CFP entered ER exit sites identified by Sec24-YFP (Fig. 2 D). In subsequent frames of the time lapse, VSVG-CFP-laden carriers repeatedly budded off from the initial point of concentration and traveled away from the exit site (Fig. 2E). After each budding event, some VSVG-CFP fluorescence remained behind and subsequently accumulated at precisely the same point (Fig. $2 E, F$ ). The oscillating changes in fluorescence corresponding to fluorescence loss during budding events and fluorescence increases during cargo accumulation are quantified in Figure $2 \mathrm{~F}$. Similar fluorescence fluctuations indicative of budding events were observed at 

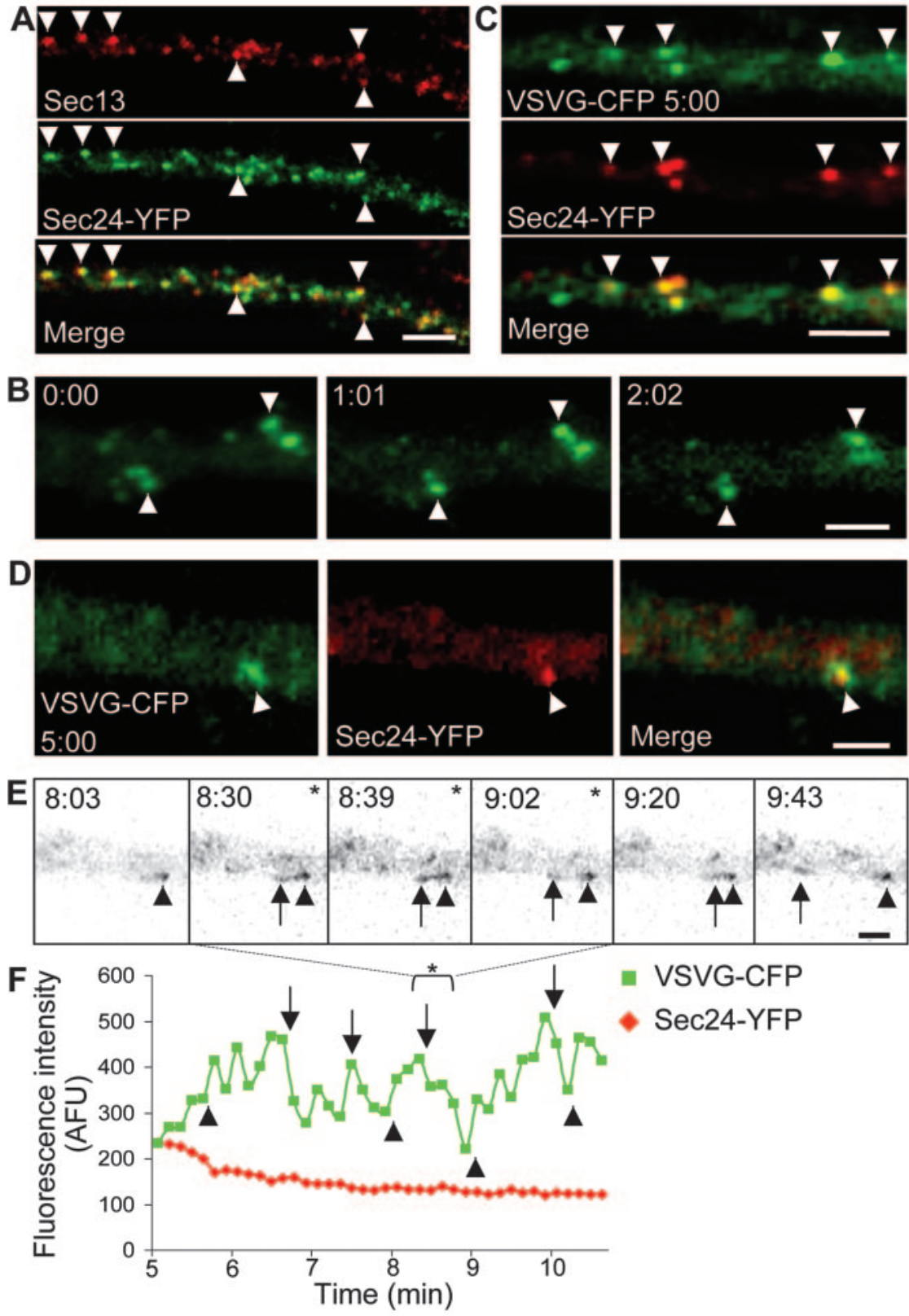

Figure 2. Functional ER exit sites are present throughout dendrites. $A$, Dendritic ER exit sites are shown either by staining for the endogenous COPII protein Sec13 (top) or by expressing Sec24-YFP (middle). Colocalizing puncta are yellow in the merged image (bottom), and representative examples are indicated by arrowheads. Scale bar, $5 \mu \mathrm{m}$. B, Sec24-YFP marks stable, stationary dendritic ER exit sites in living hippocampal neuron dendrites (15 DIV). Scale bar, $1 \mu \mathrm{m}$. See supplemental movie S4 (available at www.jneurosci.org) for time lapses. C, Five minutes after the shift to $32^{\circ} \mathrm{C}$ from the $39.5^{\circ} \mathrm{C}$ block, VSVG-CFP (top) accumulates at ER exit sites marked by Sec24-YFP (middle) in a living hippocampal neuron (14 DIV). Arrowheads mark points of colocalization. Scale bar, $2.5 \mu \mathrm{m}$. D, Five minutes after the shift from 39.5 to $32^{\circ} \mathrm{C}$, VSVG-CFP (left) accumulates at an ER exit site positive for Sec24-YFP (middle). Scale bar, $1 \mu \mathrm{m}$. E, Over the next 5 min, VSVG-CFP buds from the ER exit site shown in D. Frames showing the budding event in the VSVG-CFP channel are presented. In each frame, the arrowhead labels the stationary ER exit site, whereas the arrow labels transport vesicles budding from that site and trafficking to the left of the frame, toward the neuronal soma. Frames were acquired every 9 sec. Scale bar, $1 \mu \mathrm{m}$. See supplemental movie S5 (available at www.jneurosci.org) for time lapses. F, Quantitative analysis of VSVG-CFP and Sec24-YFP fluorescence intensity at an ER exit site. Although the Sec24-YFP fluorescence merely declines monotonically because of photobleaching (red), the VSVG-CFP fluorescence (green) fluctuates as vesicles repeatedly bud from the ER exit site and additional cargo accumulates. Loss of fluorescence because of budding events (arrows) are interleaved by periods of additional accumulation of VSVG-CFP at the site (arrowheads). The period encompassing the budding event in $E$ is marked by the asterisk. every stable ER exit site examined during the early phase (5-20 min) of secretory trafficking ( $n=5$ neurons, 20 exit sites). These data indicate that the cargo at an ER exit site does not leave in a single event, but rather is concentrated and available for multiple rounds of export as a series of carriers bud from the site. Furthermore, these experiments show that dendritic ER exit sites are stable, stationary ER subdomains that are functional components of the neuronal secretory pathway. Finally, these findings demonstrate that cargo carriers can originate at ER exit sites dispersed throughout the dendrite.

\section{Post-ER compartments in dendrites} differ in mobility and directionality An analysis of the secretory trafficking events that occurred after release of VSVG-GFP from the $39.5^{\circ} \mathrm{C}$ block revealed two phases of post-ER trafficking. Within the first $5 \mathrm{~min}$, VSVG-GFP initially present throughout the diffuse ER (Fig. $3 A$ ) accumulated at ER exit sites and budded into highly mobile carriers as described above. These carriers trafficked bidirectionally in dendrites (Fig. $1 E$ ), with a quantitative bias toward retrograde transport (Fig. 3A,C,F). Within 20 min, VSVGGFP began to accumulate within perinuclear Golgi structures in the cell soma (Fig. $3 B$ ), consistent with the trafficking kinetics of VSVG in non-neuronal cells (Presley et al., 1997; Hirschberg et al., 1998). Surprisingly, in many neurons VSVG-GFP was also present in punctate structures throughout the dendrites at times when VSVG is known to concentrate in the Golgi (20-40 min) (Presley et al., 1997) (Fig. 3B). Given that these puncta could represent either trafficking ER-to-Golgi carriers, dendritic Golgi, or possibly postGolgi transport carriers, a detailed study of these structures was performed to differentiate these populations.

Thirty-eight carriers from neurons expressing VSVG-GFP were examined during the time window from 0 to 20 min after the release from the $39.5^{\circ} \mathrm{C}$ block. These early phase carriers exhibited saltatory movement, being stationary for 15-30 sec before moving rapidly $(0.5-1.0 \mu \mathrm{m} / \mathrm{sec})$ along the dendrite. Carrier trajectory plots revealed movement in both the anterograde and retrograde directions, with quantitative bias toward retrograde transport (26 of 38 carriers moved inward) (Fig. $3 C, F)$. Plots of the lifetimes of these carriers, in which the length of the bar represents the lifetime of one carrier and the position of the bar indicates the time over which the carrier was evident, re- 

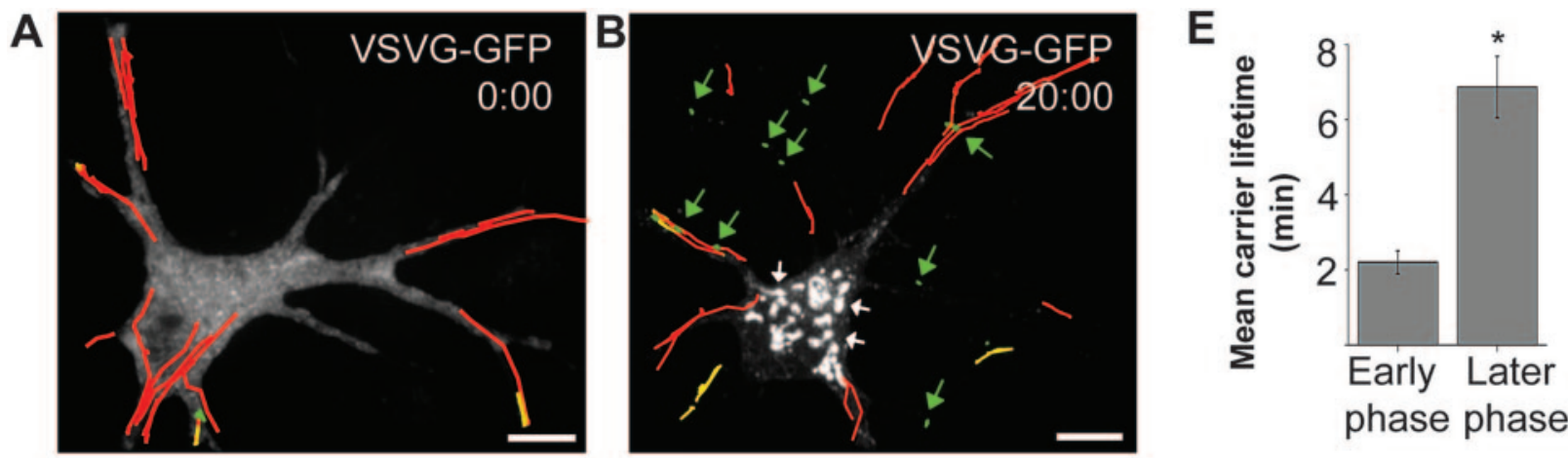

C Early phase carriers (0 to $20 \mathrm{~min}$ )

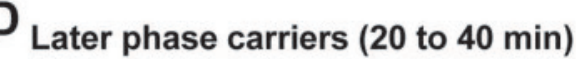

\section{$F$}
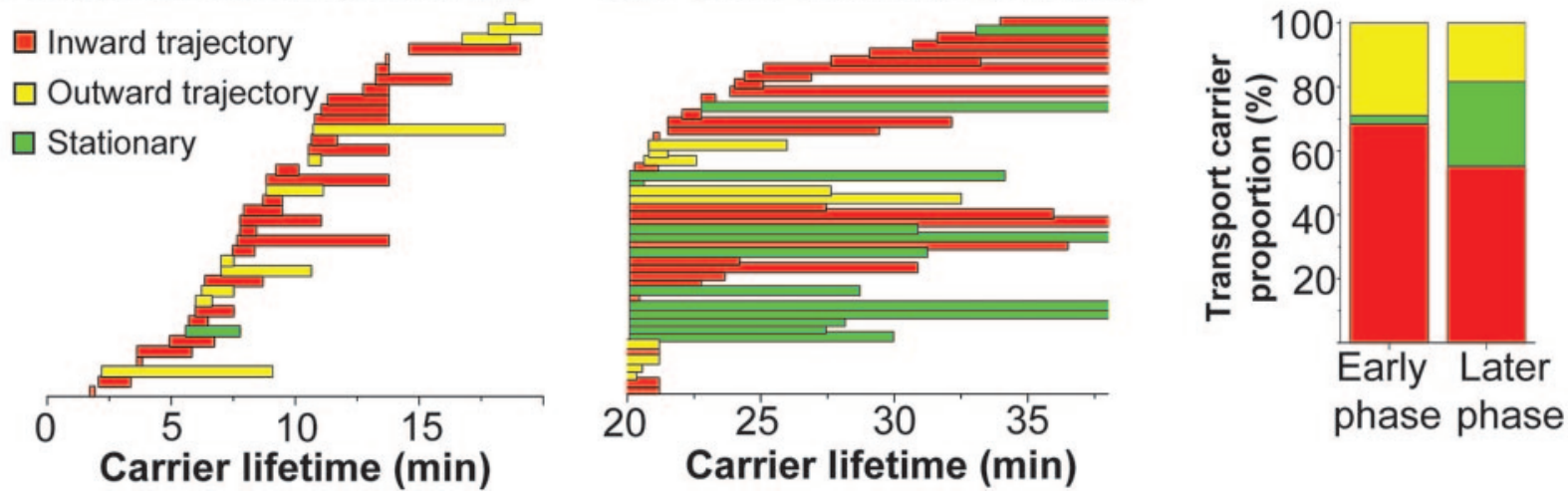

Figure 3. Rapidly transported secretory cargo accumulates at both somatic and dendritic locations after release from the ER. $A$, During the first 20 min after the release from the $39.5^{\circ} \mathrm{C} b l 0 c k$ (left), VSVG-GFP is present in vesiculotubular carriers that are highly mobile. Trajectories of moving vesicles are plotted onto an image of a hippocampal neuron. These carriers may traffic in either the retrograde (red) or anterograde direction (yellow), although in this neuron traffic is predominantly inward. In all panels, the time represents the starting point for the time lapse. Scale bar, $10 \mu \mathrm{m}$. $B$, Between 20 and $38 \mathrm{~min}$ after release from the $39.5^{\circ} \mathrm{C}$ block (right) VSVG-GFP has accumulated in stable stationary structures. These structures include perinuclear Golgi structures in the cell soma (white arrows) and more distributed small dendritic structures (green arrows). Although inwardly (red) and outwardly (yellow) moving carriers are still observed during this interval, many more stationary structures (green) are present compared with the early phase of post-ER traffic in A. Scale bar, $10 \mu \mathrm{m}$. C, Vesicle lifetime plots show that VSVG-GFP-containing structures are more transient during the early phases of ER-to-Golgi transport. Each horizontal bar represents a single dendritic VSVG-GFP-labeled structure visualized during a live imaging experiment. The length of the bar shows the period during which the VSVG-GFP-labeled structure was evident. D, Vesicle lifetime plots during the later phase of post-ER trafficking shows that between 20 and 40 min after release from $39.5^{\circ} \mathrm{C}$, VSVG-GFP is present in more stable, stationary dendritic structures. Bar color code is as in C.E, Mean carrier lifetimes at early and late phases of secretory trafficking. ${ }^{*} p<0.0001$; $t$ test. F, Proportion of VSVG carriers that are inwardly moving (red), outwardly moving (yellow), or static (green) during early and late phases of secretory trafficking. During later phases of post-ER traffic, a larger proportion of VSVG-labeled vesicles are stationary.

vealed that these carriers form and disappear throughout this early phase of post-ER trafficking (Fig. 3C). Most importantly, the lifetime of early phase carriers was short (Fig. $3 E$ ), averaging $2.2 \pm 0.2 \mathrm{~min}$, because they frequently merged with larger structures or disappeared into the much brighter neuronal soma.

The behavior of VSVG-GFP-labeled structures within the time window from 20 to $38 \mathrm{~min}$, when VSVG-GFP concentrates within the Golgi, was strikingly different from the carriers appearing earlier. In contrast to the high mobility and shorter lifetime of the early phase ER-to-Golgi carriers (Fig. 3C), later-phase structures were frequently stable and stationary for much longer periods (Fig. 3D). Although only one VSVG-GFP-labeled puncta was entirely stationary during the early phase (Fig. 3C,F), 13 of the 49 carriers followed were stationary during later intervals (Fig. 3D,F). The trajectories of these late-phase carriers revealed a widespread distribution of these stationary accumulations of VSVG-GFP throughout the dendrites (Fig. 3B). Notably, latephase VSVG-GFP carriers were also much more stable than early phase carriers. On average, the lifetime of these late-phase puncta was more than three times greater than the earlier, more highly mobile carriers $(6.5 \pm 0.5 \mathrm{~min})$ (Fig. $3 E$ ). Thus, post-ER traffic in dendrites consists of at least three kinetically distinguishable populations of vesicles/carriers that differ in both mobility and lifetime. Moreover, these data indicate that secretory cargo accumulates at both somatic and dendritic locations after release from the ER.

\section{Distributed Golgi outposts in distal dendrites}

The presence of VSVG-GFP in punctate structures throughout the dendrites at times when VSVG-GFP is known to accumulate in the Golgi (Presley et al., 1997) (see also Fig. 3B) raised the possibility that these structures represent dendritic Golgi. We investigated whether dendrites contain Golgi by staining for the endogenous Golgi matrix protein GM130 (Fig. 4A) (Nakamura et al., 1995). In all neurons, GM130 was present as a convoluted complex in the cell soma (Fig. $4 A$, top). In many cells, we also saw GM130 in the proximal dendrite (Fig. $4 A$, top), either as an obvious extension of somatic Golgi or as a seemingly discrete Golgi fragment. This result is in agreement with previous immunocytochemical data identifying Golgi structures in proximal neuronal dendrites (Krijnse-Locker et al., 1995; Torre and Steward, 1996). However, we also found that in many neurons, GM130 marked structures much farther out in dendrites. Bright GM130- 

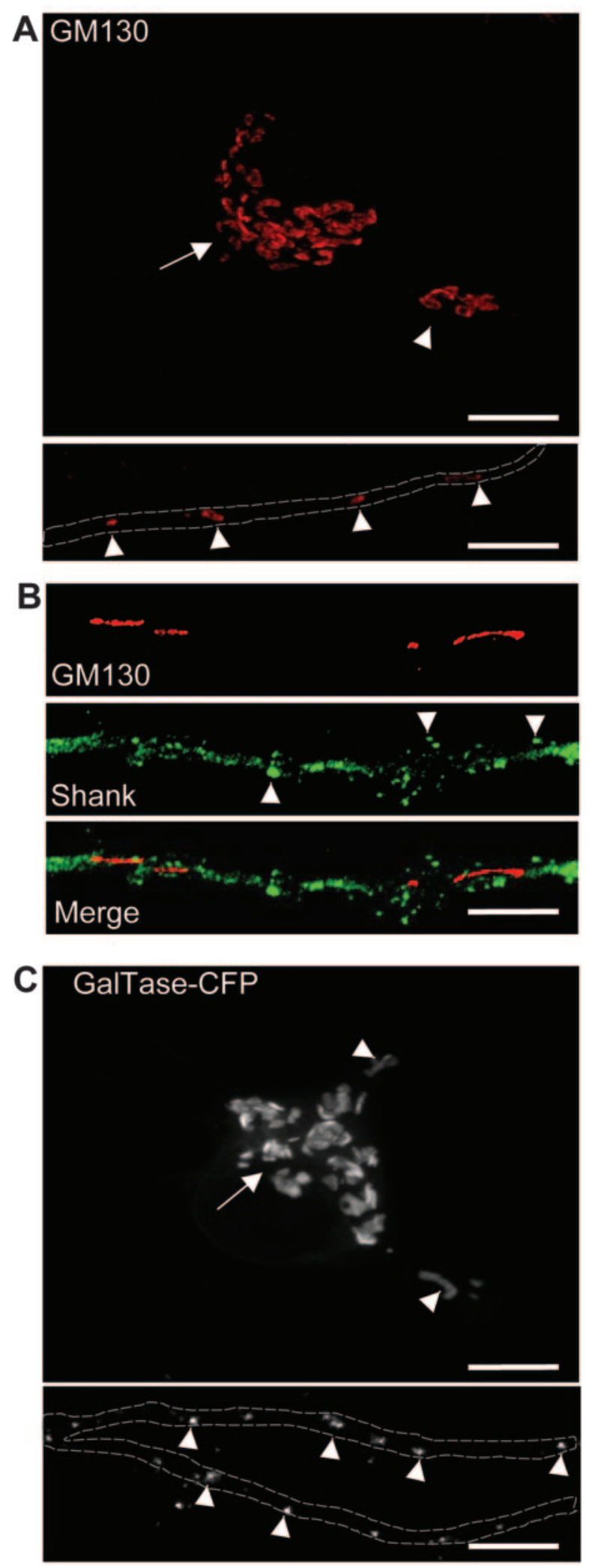

Figure 4. Distributed Golgi outposts in distal dendrites. A, Staining for GM130 reveals the presence of Golgi structures within the neuronal soma (top, arrow) and immediately proximal dendrite (top, arrowhead) (14 DIV). Examination of distal dendrites revealed numerous tubular and punctate GM130-positive structures (lower, arrowheads). B, Dendritic Golgi labeled for GM130 (red) do not colocalize with Shank (green). C, Expression of GalTase-CFP shows the distribution of Golgi in the soma (top, arrow), proximal dendrite (top, arrowheads), and distal dendrite (bottom, arrowheads) in a living 14 DIV hippocampal neuron. Scale bars, $5 \mu \mathrm{m}$. labeled structures were frequently present up to $100 \mu \mathrm{m}$ from the neuronal soma and appeared to be discontinuous from somatic Golgi (Fig. $4 A$, bottom, $B$, top). These structures varied in shape from oblong to more punctate, and ranged in size between 1 and $4 \mu \mathrm{m}$ in diameter These structures were usually located in the dendritic shafts, with no obvious relationship to excitatory synapses on dendritic spines, as marked by staining for the synaptic protein Shank (Fig. 4B).

To test whether the distributed outposts of Golgi matrix in dendrites were associated with enzyme-containing Golgi membranes, we expressed galactosyltransferase, a well characterized resident Golgi enzyme, tagged with cyan fluorescent protein (GalTase-CFP) (Cole et al., 1996). Like GM130, GalTase-CFP marked a convoluted Golgi in the cell soma, and frequently labeled structures in the immediately proximal dendrites (Fig. 4C, top). However, much like GM130, GalTase-CFP was also present as distributed punctate structures in dendrites of a subset of neurons (Fig. $4 C$, bottom). These structures were most common in the proximal dendrites, but were also present more distally in secondary and tertiary dendrites. In sharp contrast, no Golgi structures were observed in axons (data not shown). Together, these data provide strong evidence for discrete, discontinuous, distributed Golgi elements in distal dendrites.

\section{Dendritic Golgi outposts receive early secretory cargo}

Small Golgi fragments are sufficient to produce a functional secretory system (Pelletier et al., 2000). To determine the functionality of Golgi outposts in dendrites, we expressed VSVG-GFP in hippocampal neurons and allowed forward trafficking for $20 \mathrm{~min}$ at a permissive temperature. As above (Fig. 3B), by 20 min VSVGGFP accumulated into stable, stationary compartments in neuronal dendrites (Fig. 5A). In all cases, these dendritic VSVG-GFP compartments colocalized with the Golgi matrix protein GM130 (Fig. 5A). Moreover, colocalization of VSVG with Golgi markers was also observed in living neurons expressing both VSVG-YFP $\left(20 \mathrm{~min}\right.$ at $32^{\circ} \mathrm{C}$ ) and GalTase-CFP (Fig. 5B).

To visualize cargo transport into dendritic Golgi directly, twocolor time-lapse imaging was performed on hippocampal neurons expressing VSVG-YFP and GalTase-CFP. After prolonged incubation at $39.5^{\circ} \mathrm{C}$, VSVG-YFP localized to diffuse distributed ER in dendrites, whereas GalTase-CFP labeled numerous round or elongated Golgi outposts throughout the dendritic length (Fig. $5 C)$. After $20 \mathrm{~min}$ at $32^{\circ} \mathrm{C}$, the distribution of GalTase-CFP was essentially unchanged (Fig. $5 D$, bottom, arrows). However, the distribution of VSVG-YFP changed dramatically over this period, becoming concentrated in the structures labeled positive for GalTase-CFP (Fig. 5D, top, arrows). Furthermore, rapid timelapse imaging frequently captured the merging of mobile VSVGladen transport carriers with stationary collections of cargo indicative of dendritic Golgi (Fig. 5E).

The time-dependent increase in colocalization between newly released VSVG-YFP and GalTase-CFP was evident when the fluorescence intensity along the length of the dendrite was plotted for each fluorophore (Fig. 5F,G). Initially, there was little coincidence of intensity peaks for VSVG-YFP and GalTase-CFP (Fig. $5 F$ ). However, 30 min later, VSVG-YFP fluorescence appeared in peaks threefold to fourfold more intense than the diffuse pattern at time 0 (Fig. $5 G$ ), indicating that VSVG-YFP had accumulated into specific dendritic regions over time. A comparison of the VSVG-YFP and GalTase-CFP plots after $20 \mathrm{~min}$ showed that nearly every point of VSVG-YFP accumulation corresponded to a position along the dendrite at which GalTase-CFP was localized (Fig. 5G). Spatial pixel intensity correlation plots revealed that, 
A

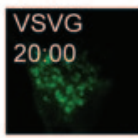

GM130

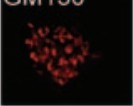

Merge

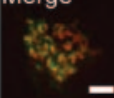

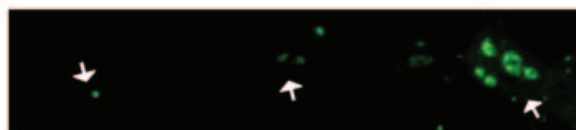
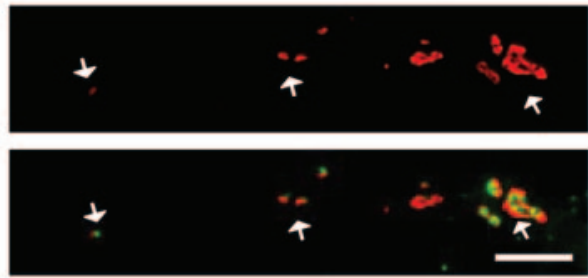

VSVG-YFP

0:00

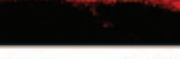

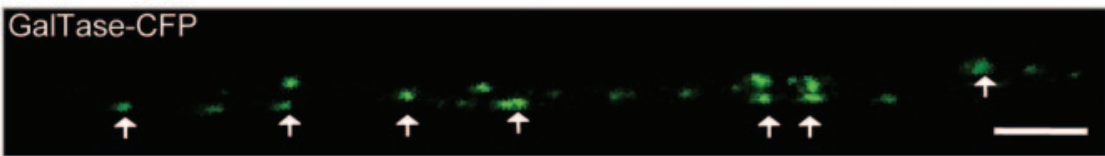

VSVG-YFP

20:00

20:00

个

GalTase-CFP

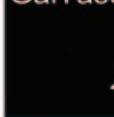

†
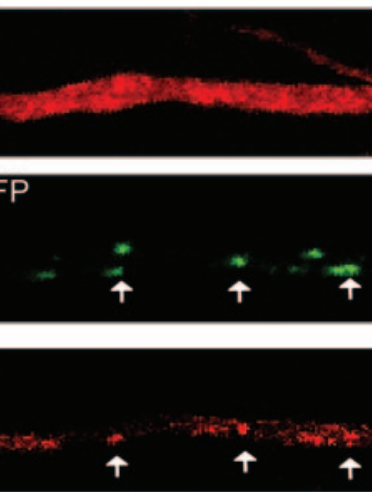

$\uparrow$
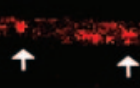

$\uparrow$
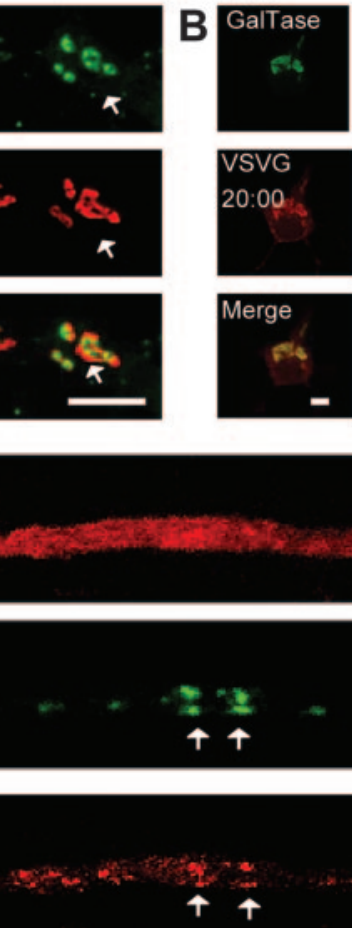
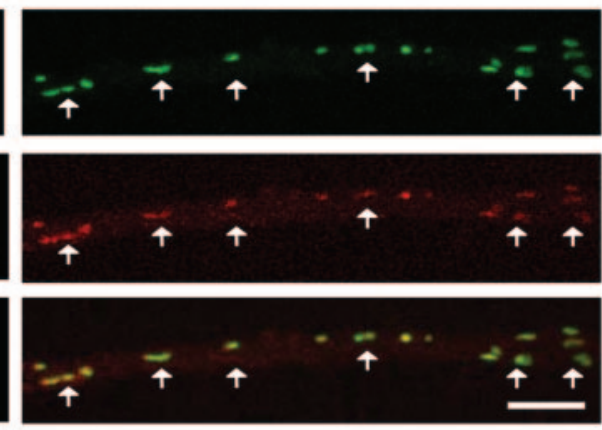

E
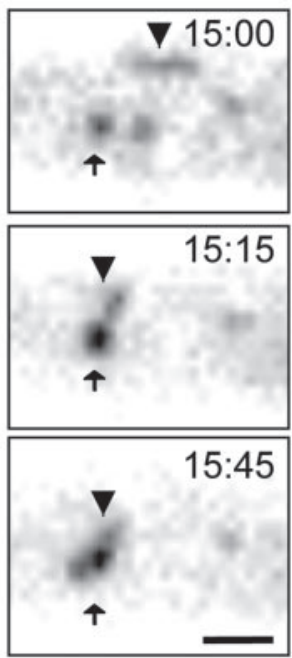

F

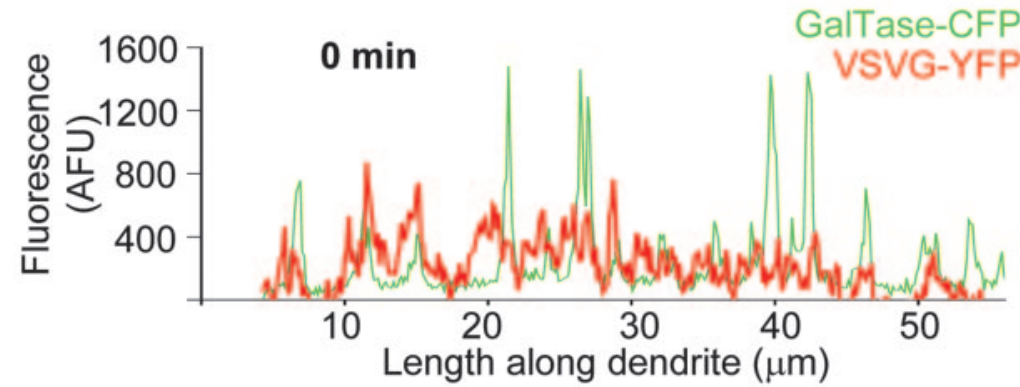

G

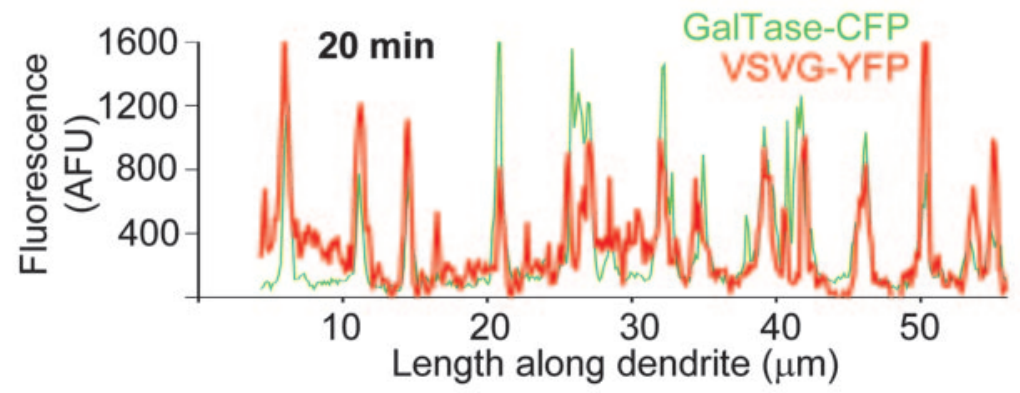

$\mathrm{H}$

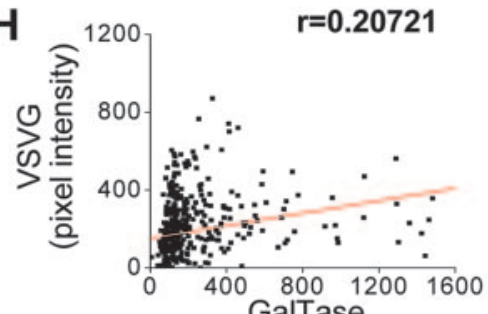

GalTase

(pixel intensity)

I

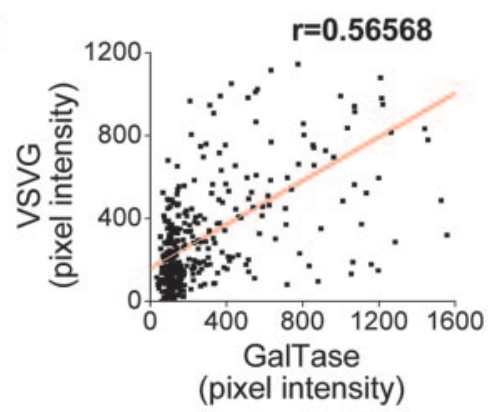

Figure 5. Dendritic Golgi function in early secretory trafficking. A, Twenty minutes after release from $39.5^{\circ} \mathrm{C}$ block, VSVG-GFP (green) collects into fragmented Golgi in both the soma and the dendrites of expressing hippocampal neurons (14 DIV) (top). Both somatic (left) and dendritic (right, arrows) Golgi stain for GM130 (red). Scale bars, $5 \mu$ m. B, A live neuron (17 DIV) expressing both VSVG-YFP (red) and GalTase-CFP (green) imaged $20 \mathrm{~min}$ after the release from the $39.5^{\circ} \mathrm{C}$ block shows VSVG-YFP present in somatic (left) and dendritic (right) structures positive for GalTase-CFP. Scale bars, $5 \mu \mathrm{m}$. C, Immediately after the $39.5^{\circ} \mathrm{C}$ block, VSVG-YFP (red) is distributed diffusely throughout the ER of the dendrite of a living hippocampal neuron (15 DIV). GalTase-CFP (green) marks punctate dendritic Golgi outposts (arrows) in the same neuron. Scale bar, $5 \mu \mathrm{m}$. D, The same dendrite as in ( imaged 20 min after the release from the $39.5^{\circ} \mathrm{C}$ block. At this point, VSVG-YFP (red) has accumulated in GalTase-CFP-labeled structures (green). Note that the positions of dendritic Golgi outposts (arrows), as marked by GalTase-CFP, remain in a relatively fixed position over the 20 min. $E$, Time-lapse frames illustrating fusion of a mobile post-ER carrier (arrowhead) with a stationary dendritic Golgi outpost (arrow). Scale bar, $1 \mu \mathrm{m}$. See supplemental movie $\mathbf{S 6}$ (available at www.jneurosci.org) for time lapses. F, Line-scan analysis along the dendrite shows no initial spatial relationship between VSVG-YFP (red) and GalTase-CFP ( $g r e e n)$ at 0 min after the release from the $39.5^{\circ} \mathrm{C}$ block. $\mathrm{G}$, Line-scan analysis $20 \mathrm{~min}$ after the release from the $39.5^{\circ} \mathrm{C}$ block shows that regions of accumulated VSVG-YFP fluorescence (red peaks) correspond to regions of GalTase-CFP fluorescence (green peaks). $H$, Correlation plot of VSVG-YFP and GalTase-CFP pixel values shows little correlation between initial distributions of fluorescence ( 0 min). The linear fits to the data (red line) and the correlation coefficients ( $r$ ) are shown in $H$ and $I$. I, Twenty minutes later, VSVG-YFP and GalTase-CFP fluorescence values are much more highly correlated as VSVG-YFP traffics into GalTase-CFP labeled structures. 

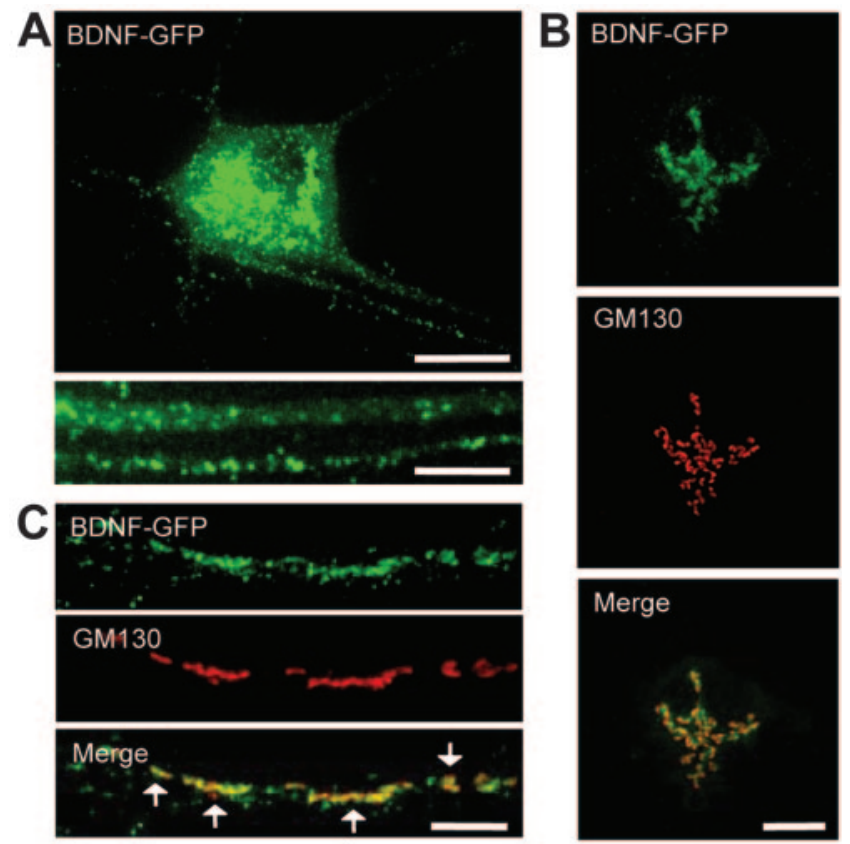

Figure 6. BDNF-GFP traffics to somatic and dendritic Golgi. A, BDNF-GFP expressed in a hippocampal neuron (15 DIV). BDNF-GFP is present throughout the neuronal soma (top) and dendrites (bottom) in punctate vesicular structures. B, After $3 \mathrm{hr}$ at $20^{\circ} \mathrm{C}$, BDNF-GFP (green) has accumulated into somatic Golgi structures positive for GM130 (red). C, Incubation at $20^{\circ} \mathrm{C}$ also causes accumulation of BDNF-GFP into dendritic Golgi, identified by staining for GM130. Areas of colocalization are indicated by arrows. Scale bars, $10 \mu \mathrm{m}$.

although initially little correlation existed between VSVG-YFP and GalTase-CFP pixel intensity values (Fig. $5 \mathrm{H}$ ), after $20 \mathrm{~min}$ the correlation between the two proteins increased significantly (Fig. 5I). Finally, although the relative sizes of the GalTase-CFP peaks varied somewhat over time, the position of these peaks did not (Fig. 5, compare $F$ and $G$ ). This is consistent with the observed stationary nature of dendritic Golgi over time (Fig. 5C,D). In summary, these findings demonstrate that the requisite machinery exists for ER-to-Golgi trafficking in neuronal dendrites and that this machinery is competent for cargo transport.

The secreted neurotrophic factor BDNF uses both somatic and dendritic Golgi

The dendritic release of soluble growth factors is thought to mediate local signaling and morphological regulation (Yacoubian and Lo, 2000; Danzer et al., 2002; Horch and Katz, 2002). Having shown that the integral membrane protein VSVG-GFP is trafficked to both somatic and dendritic Golgi (Fig. 5), we sought to determine whether secreted proteins also use dual modes of ERto-Golgi transport. To this end, we expressed a GFP fusion of the brain-derived neurotrophic factor (BDNF) (Egan et al., 2003) and examined ER-to-Golgi trafficking in hippocampal neurons. At steady state, BDNF-GFP is present throughout the neuronal soma and dendrites, where it occupies punctate vesicular structures (Fig. 6A). To accumulate newly synthesized BDNF-GFP in the neuronal Golgi, we used a $20^{\circ} \mathrm{C}$ temperature block, which prevents budding of cargo from the Golgi (Matlin and Simons, 1983). After a $3 \mathrm{hr}$ incubation at $20^{\circ} \mathrm{C}$, BDNF-GFP showed extensive colocalization with GM130 in the neuronal soma (Fig. $6 \mathrm{~B}$ ), as well as in Golgi outposts in distal dendrites (Fig. 6C). These data show that, as with integral membrane proteins, se-
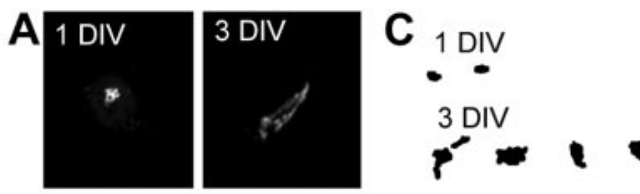

$10 \underline{\mu \mathrm{m}}$
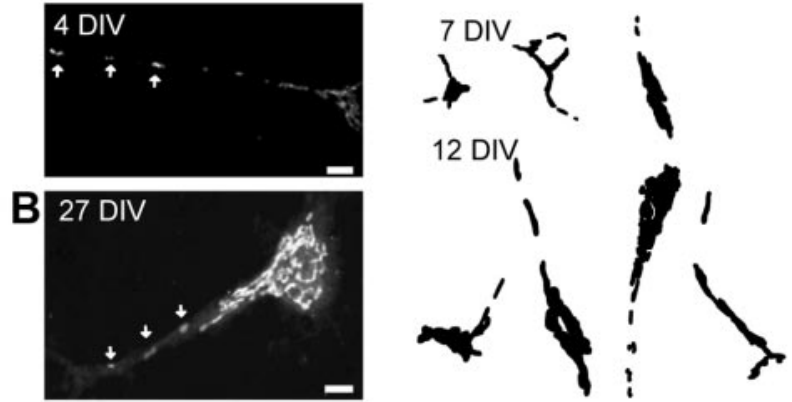

D
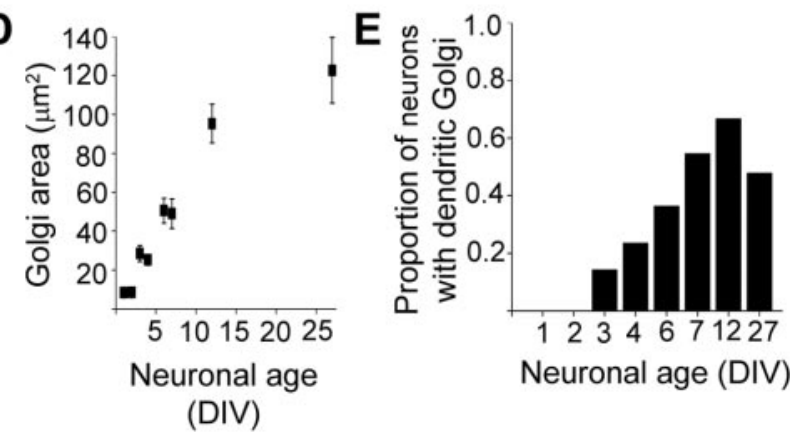

Figure 7. Expansion and partitioning of the Golgi during neuronal development. A, Golgi visualized by staining for GM130 in neurons at early stages of development. At 1 DIV the Golgi complex is small, compact, and exclusively somatic, but subsequently grows along with the neuron (3 DIV). By 4 DIV, Golgi extends far into the dendrites as discrete Golgi outposts (bottom, arrows). Scale bar, $5 \mu \mathrm{m}$. B, Golgi in a mature neuron (27 DIV) marked by staining for GM130. Note the presence of dendritic Golgi outposts (arrows). Scale bar, $5 \mu \mathrm{m}$. C, Traces of Golgi, labeled by GM130 staining, from neurons of various ages shows the developmentally regulated growth and extension of Golgi into proximal and middle neuronal dendrites. Distal Golgi structures are present but not shown here because of the small size and scale. Scale bar, $5 \mu \mathrm{m} . D$, Quantitative analysis of Golgi growth during neuronal maturation. Data are means \pm SEM of Golgi area ( $n=130$ total neurons, with at least 11 for each time point). Normalized histogram of the proportion of neurons with dendritic Golgi during neuronal maturation.

creted proteins use both somatic and distributed dendritic secretory elements.

Developmental expansion and partitioning of neuronal Golgi Although distal dendritic Golgi outposts were present in a subset of neurons, they were not a universal phenomenon in all neurons or all dendrites. To determine the origin of dendritic Golgi outposts, we performed a study of Golgi morphology in developing neurons by staining for GM130 in cultured neurons of various ages in vitro. The growth of neurons in culture is a well described phenomenon and is known to proceed through stages analogous to neuronal growth in vivo (Dotti et al., 1988). At very early stages of development [1-2 d in vitro (DIV)], neurons have not yet begun to extend processes. At this stage, neuronal Golgi were present as single small $\left(\sim 9 \mu \mathrm{m}^{2}\right)$ perinuclear structures with a compact morphology (Fig. $7 A$, top left, $C, D$ ), very similar to the Golgi complex of typical non-neuronal cell types (Shorter and Warren, 2002). During the subsequent period of neuronal growth and differentiation, the Golgi underwent marked expansion (Fig. 7A-D). Quantitative morphometry revealed a 10 -fold increase in Golgi apparatus size over the first 12 DIV (Fig. 7D), at which time the Golgi reached its mature mor- 


\section{Dual modes of ER-to-Golgi transport:}

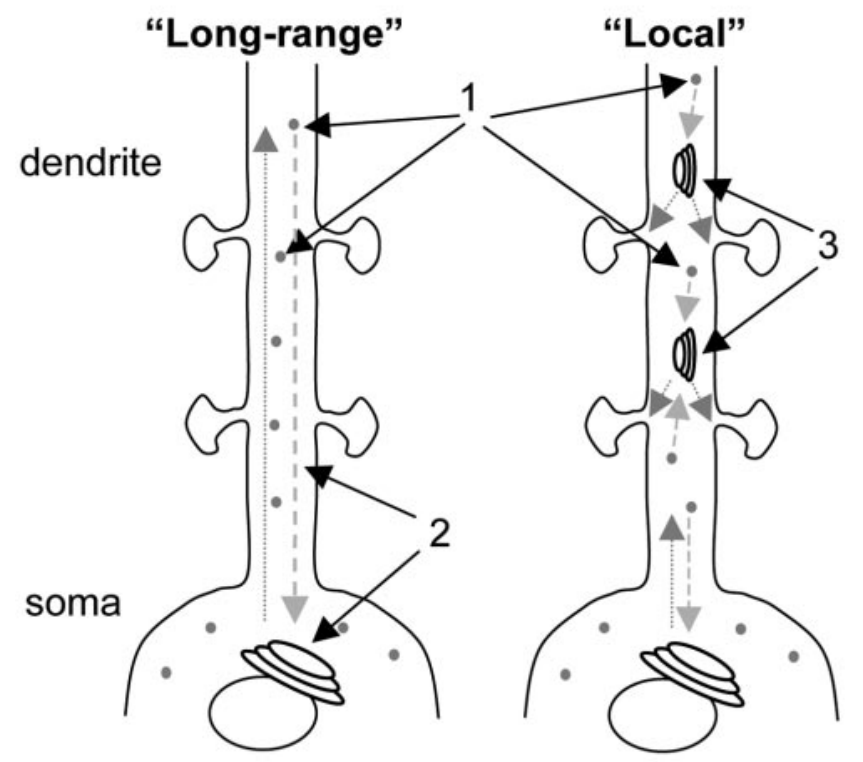

- ER exit sites

@ Golgi

$\rightarrow$ Pre-Golgi traffic

Post-Golgi traffic

Figure 8. Proposed model of ER-to-Golgi trafficking in neuronal dendrites. An illustration of the dual organization of early secretory pathway components in neuronal dendrites. See text for details.

phology (Fig. 7C). Interestingly, dendritic Golgi outposts first appeared after 3 DIV and steadily increased up to 12 DIV, a time at which neurons are undergoing rapid process outgrowth and synaptogenesis (Dotti et al., 1988). In some cells, the dendritic extension was obviously a continuation of somatic Golgi. However, in other neurons, the dendritic Golgi appeared as discrete fragments discontinuous from the neuronal soma (Fig. $7 A$, bottom, $C$ ). In mature neurons (27 DIV), the large surface area, as well as the dendritic Golgi outposts, were maintained (Fig. $7 B, E$ ). Golgi structure and distribution was unaffected by the presence or absence of serum or contacting astrocytes (data not shown). Together, these results demonstrate a marked expansion and partitioning of the Golgi apparatus during neuronal development, and suggest that spatial restriction of secretory organelles occurs as dendrites differentiate.

\section{Discussion}

Here we report dual modes of early secretory traffic in neurons. Figure 8 summarizes these results in a model of the neuronal secretory pathway. (1) In all neurons, cargo buds from the ER at specialized ER exit sites, located in both the soma and dendrites, and traffics to the neuronal Golgi. (2) In a subset of neurons, Golgi is present only in the neuronal soma, necessitating longrange inward trafficking of pre-Golgi carriers (long-range ER-toGolgi transport). (3) Other neurons have, in addition to somatic Golgi, dendritic Golgi structures that function in secretory trafficking (local ER-to-Golgi transport). Dendritic ER exit sites and Golgi compartments function in secretory trafficking in parallel with the somatic Golgi, and are thus positioned to serve particular dendritic regions or sets of synapses.

\section{Dendritic Golgi outposts are novel components of the secretory pathway}

A principal finding from the current study is the observation that many neurons possess both somatic Golgi and discrete, discontinuous Golgi-type structures located far into the dendrites. These dendritic Golgi outposts possess both matrix and enzyme components, and function similarly to the somatic Golgi in the transport of VSVG and BDNF. In other mammalian cells, the Golgi consists of a series of interconnected, flattened cisternae that are located in the perinuclear region (Rambourg and Clermont, 1990). Here we have shown that this core organization is present in neurons, but, in addition, many neurons possess discrete packets of Golgi throughout neuronal dendrites. This distributed network of secretory organelles may allow for more localized control over protein sorting and membrane composition than would be conferred if Golgi were concentrated solely in the cell body. Dendritic Golgi outposts are much smaller, at 1-4 $\mu \mathrm{m}$ across, than somatic Golgi, which may have a surface area of tens of micrometers. It is possible that, given their small size, dendritic outposts are specialized to process only a subset of neuronal proteins. However, as only a minute fraction of Golgi is necessary to restore full secretory capability in transected cytoplasts (Pelletier et al., 2000), it seems likely that dendritic Golgi outposts provide dendrites with substantial secretory capacity.

Previous ultrastructural studies have reported membranes analogous to Golgi cisternae within the spines of distal dendrites (Pierce et al., 2000, 2001). In our experiments, we observed dendritic Golgi exclusively within dendritic shafts. Several possibilities exist for this difference. Because our experiments are at the light microscopic level, it is possible that minute Golgi fragments beyond the limits of resolution are present in dendritic spines. Alternatively, because Golgi membrane and proteins are known to cycle between the ER and Golgi (Cole et al., 1998), membranes labeled for markers of these compartments in spines could represent carriers cycling between the ER and Golgi rather than core Golgi. In any event, whether in spines or shafts or both, the dendritic localization of early secretory compartments strongly supports localized biosynthetic production of membrane and membrane proteins near postsynaptic compartments.

Elaboration of a dendritic arbor and extension of an axon define neuronal shape and are the key morphological features of neuronal maturation (Bradke and Dotti, 2000; Jan and Jan, 2001; Scott and Luo, 2001). Both processes involve large increases in the surface area of the plasma membrane that require selective targeting of integral membrane proteins (Higgins et al., 1997, Burack et al., 2000; Sampo et al., 2003) as well as massive production of lipid membranes and surface incorporation via exocytosis (Bradke and Dotti, 2000; Martinez-Arca et al., 2001). Our findings indicate that this period of process extension and surfacearea expansion is accompanied by marked growth of the neuronal Golgi and fragmentation into dendritic Golgi outposts. In non-neuronal cells, such as migrating fibroblasts (Kupfer et al., 1983) and budding yeast (Finger and Novick, 1998), the spatial orientation of secretory organelles can direct exocytosis for specific membrane domains. Expansion of the Golgi-associated secretory apparatus in developing neurons may similarly be polarized to accommodate the increased demand for surface membrane during process outgrowth. Moreover, expansion and dispersal of secretory organelles may regulate neuronal morphology. In axons, new membrane is added at the tips of extending 
processes (Craig et al., 1995; Zakharenko and Popov, 1998). In growing dendrites, the points of membrane addition are uncertain, but the highly branched nature of dendrites suggests a requirement for multiple independently regulated points of membrane addition (Bradke and Dotti, 2000), a requirement ideally suited by autonomous dendritic secretory systems.

\section{The distribution of neuronal Golgi is regulated by development}

Here we have shown that dendritic Golgi outposts appear during early periods of neuronal differentiation. Golgi outposts may form as fragments derived from somatic Golgi that expand into dendrites as the dendrites themselves grow. Alternatively, Golgi outposts may form de novo from transitional ER elements in a manner similar to that observed in the unicellular yeast Pichia pastoris (Rossanese et al., 1999; Bevis et al., 2002). In either case, it seems quite likely that specific signals locally control the formation, distribution, and abundance of dendritic Golgi. During cell division, perinuclear Golgi in non-neuronal cells undergoes characteristic fragmentation induced by mitotic kinases (Acharya et al., 1998; Kano et al., 2000; Sutterlin et al., 2001; Shorter and Warren, 2002; Colanzi et al., 2003). Similarly, in response to apoptotic stimuli, the Golgi apparatus fragments into small vesiculotubular elements (Sesso et al., 1999). In both cases, fragmentation involves post-translational modification and reorganization of Golgi matrix proteins (Dirac-Svejstrup et al., 2000; Chiu et al., 2002; Lane et al., 2002). Perhaps similar signaling cascades are also operating in the neuronal setting to regulate Golgi distribution. In this light, it is interesting to note that signaling pathways including ERK-MAPK (extracellular signal-regulated kinasemitogen-activated protein kinase) and Rho-family GTPases, which play a central role in regulating dendritic morphology (Scott and Luo, 2001; Wu et al., 2001; Li et al., 2002; Vaillant et al., 2002), likewise exert profound effects on Golgi structure and fragmentation in non-neuronal cells (Acharya et al., 1998; Nobes and Hall, 1999). It will be important for future studies to determine the signals and regulatory mechanisms that control Golgi distribution in dendrites.

\section{Secretory organelles and dendritic synthesis of membrane proteins and secreted factors}

Much attention has been given to the possibility of local protein synthesis as a mechanism for synapse-specific modifications underlying synaptic plasticity (Jiang and Schuman, 2002; Steward and Worley, 2002). Although local synthesis of cytosolic proteins requires only mRNA and ribosomes, the production and processing of integral membrane proteins requires additional elements, including ER, Golgi, and associated vesicular transport. The visualization of cargo proceeding through the dendritic secretory pathway in the current study establishes that many neuronal dendrites have all the functional components required for processing newly synthesized membrane proteins. Consistent with this notion, isolated dendrites are capable of incorporating sugar precursors indicative of Golgi-like function (Torre and Steward, 1996), and immunogold reactivity for protein components of secretory organelles has been observed on internal dendritic endomembranes (Pierce et al., 2000, 2001). Furthermore, we have shown that both long-range and local modes of ER-to-Golgi trafficking are used by secreted proteins, specifically the secreted growth factor BDNF. Dendritic processing of BDNF is consistent with the known effect of local BDNF overproduc- tion and TrkB activation on the growth and branching of nearby dendrites (Yacoubian and Lo, 2000; Danzer et al., 2002; Horch and Katz, 2002) and observations that neurites release BDNF in response to activity (Kojima et al., 2001). Our results provide direct evidence that proper ER-to-Golgi trafficking of locally translated proteins is possible in a subset of dendrites, events necessary to confer onto dendrites the synthetic capacity for integral membrane proteins and secreted growth factors.

\section{Long-range secretory trafficking to and from the soma}

A remarkable feature of the current study is that, although many neurons possess components of the secretory pathway allowing dendritic ER-to-Golgi trafficking autonomous of the neuronal soma, other neurons have a Golgi compartment that is entirely somatic. In these neurons, all cargo exiting the ER in the dendrites must traffic in a manner retrograde to the somatic Golgi. Indeed, even in neurons possessing dendritic Golgi outposts, the bulk of ER-to-Golgi traffic is long-range toward the soma. This raises important questions about protein targeting in these neurons and suggests that pre-Golgi carriers retain specific identities over long distances. In other cells, chaperone proteins cycling between the ER and Golgi may fulfill this function (Klumperman, 2000), and it is tempting to speculate that neurons possess specialized versions or variants of the coat proteins, chaperones, Rab-family GTPases, or soluble $N$-ethylmaleimide-sensitive factor (NSF) attachment protein (SNAP) receptors that specify pre-Golgi vesicle identity. Furthermore, we have found that, although some dendritic pre-Golgi carriers merge with dendritic Golgi outposts, others bypass these outposts en route to the somatic Golgi, suggesting that pre-Golgi carriers themselves exist in distinct populations. Given the involvement of secretory trafficking in many aspects of neuronal function, including polarity establishment and morphogenesis, synapse formation, and synaptic plasticity (Craig et al., 1994; Kirsch, 1999; Song and Poo, 1999; Bradke and Dotti, 2000; Sheng and Pak, 2000), it will be important for future studies to delineate the mechanisms that establish and regulate dendritic secretory organelles, and the cellular consequences of this unanticipated dual organization of ER-to-Golgi trafficking.

\section{References}

Acharya U, Mallabiabarrena A, Acharya JK, Malhotra V (1998) Signaling via mitogen-activated protein kinase kinase $(\mathrm{MEK} 1)$ is required for Golgi fragmentation during mitosis. Cell 92:183-192.

Antonny B, Schekman R (2001) ER export: public transportation by the COPII coach. Curr Opin Cell Biol 13:438-443.

Baumann O, Walz B (2001) Endoplasmic reticulum of animal cells and its organization into structural and functional domains. Int Rev Cytol 205:149-214.

Bergmann JE (1989) Using temperature-sensitive mutants of VSV to study membrane protein biogenesis. Methods Cell Biol 32:85-110.

Bevis BJ, Hammond AT, Reinke CA, Glick BS (2002) De novo formation of transitional ER sites and Golgi structures in Pichia pastoris. Nat Cell Biol 4:750-756.

Bradke F, Dotti CG (2000) Establishment of neuronal polarity: lessons from cultured hippocampal neurons. Curr Opin Neurobiol 10:574-581.

Burack MA, Silverman MA, Banker G (2000) The role of selective transport in neuronal protein sorting. Neurol 26:465-472.

Chiu R, Novikov L, Mukherjee S, Shields D (2002) A caspase cleavage fragment of p115 induces fragmentation of the Golgi apparatus and apoptosis. J Cell Biol 18:18.

Colanzi A, Sutterlin C, Malhotra V (2003) RAF1-activated MEK1 is found on the Golgi apparatus in late prophase and is required for Golgi complex fragmentation in mitosis. J Cell Biol 161:27-32. 
Cole NB, Smith CL, Sciaky N, Terasaki M, Edidin M, Lippincott-Schwartz J (1996) Diffusional mobility of Golgi proteins in membranes of living cells. Science 273:797-801.

Cole NB, Ellenberg J, Song J, DiEuliis D, Lippincott-Schwartz J (1998) Retrograde transport of Golgi-localized proteins to the ER. J Cell Biol 140:1-15.

Craig AM, Wyborski RJ, Banker G (1995) Preferential addition of newly synthesized membrane protein at axonal growth cones. Nature 375:592-594.

Danzer SC, Crooks KR, Lo DC, McNamara JO (2002) Increased expression of brain-derived neurotrophic factor induces formation of basal dendrites and axonal branching in dentate granule cells in hippocampal explant cultures. J Neurosci 22:9754-9763.

Dirac-Svejstrup AB, Shorter J, Waters MG, Warren G (2000) Phosphorylation of the vesicle-tethering protein $\mathrm{p} 115$ by a casein kinase II-like enzyme is required for Golgi reassembly from isolated mitotic fragments. J Cell Biol 150:475-488.

Dotti CG, Sullivan CA, Banker GA (1988) The establishment of polarity by hippocampal neurons in culture. J Neurosci 8:1454-1468.

Egan MF, Kojima M, Callicott JH, Goldberg TE, Kolachana BS, Bertolino A, Zaitsev E, Gold B, Goldman D, Dean M, Lu B, Weinberger DR (2003) The BDNF val66met polymorphism affects activity-dependent secretion of BDNF and human memory and hippocampal function. Cell 112:257-269.

Finger FP, Novick P (1998) Spatial regulation of exocytosis: lessons from yeast. J Cell Biol 142:609-612.

Futerman AH, Banker GA (1996) The economics of neurite outgrowththe addition of new membrane to growing axons. Trends Neurosci 19:144-149.

Hammond AT, Glick BS (2000) Dynamics of transitional endoplasmic reticulum sites in vertebrate cells. Mol Biol Cell 11:3013-3030.

Higgins D, Burack M, Lein P, Banker G (1997) Mechanisms of neuronal polarity. Curr Opin Neurobiol 7:599-604.

Hirschberg K, Miller CM, Ellenberg J, Presley JF, Siggia ED, Phair RD, Lippincott-Schwartz J (1998) Kinetic analysis of secretory protein traffic and characterization of golgi to plasma membrane transport intermediates in living cells. J Cell Biol 143:1485-1503.

Hong W (1998) Protein transport from the endoplasmic reticulum to the Golgi apparatus. J Cell Sci 111:2831-2839.

Horch HW, Katz LC (2002) BDNF release from single cells elicits local dendritic growth in nearby neurons. Nat Neurosci 5:1177-1184.

Jan YN, Jan LY (2001) Dendrites. Genes Dev 15:2627-2641.

Jiang C, Schuman EM (2002) Regulation and function of local protein synthesis in neuronal dendrites. Trends Biochem Sci 27:506-513.

Kacharmina JE, Job C, Crino P, Eberwine J (2000) Stimulation of glutamate receptor protein synthesis and membrane insertion within isolated neuronal dendrites. Proc Natl Acad Sci USA 97:11545-11550.

Kano F, Takenaka K, Yamamoto A, Nagayama K, Nishida E, Murata M (2000) MEK and Cdc2 kinase are sequentially required for Golgi disassembly in MDCK cells by the mitotic Xenopus extracts. J Cell Biol 149:357-368.

Kirchhausen T (2000) Three ways to make a vesicle. Nat Rev Mol Cell Biol 1:187-198.

Kirsch J (1999) Assembly of signaling machinery at the postsynaptic membrane. Curr Opin Neurobiol 9:329-335.

Klumperman J (2000) Transport between ER and Golgi. Curr Opin Cell Biol 12:445-449.

Kojima M, Takei N, Numakawa T, Ishikawa Y, Suzuki S, Matsumoto T, Katoh-Semba R, Nawa H, Hatanaka H (2001) Biological characterization and optical imaging of brain-derived neurotrophic factor-green fluorescent protein suggest an activity-dependent local release of brainderived neurotrophic factor in neurites of cultured hippocampal neurons. J Neurosci Res 64:1-10.

Krijnse-Locker J, Parton RG, Fuller SD, Griffiths G, Dotti CG (1995) The organization of the endoplasmic reticulum and the intermediate compartment in cultured rat hippocampal neurons. Mol Biol Cell 6:1315-1332.

Kuehn MJ, Schekman R (1997) COPII and secretory cargo capture into transport vesicles. Curr Opin Cell Biol 9:477-483.

Kupfer A, Dennert G, Singer SJ (1983) Polarization of the Golgi apparatus and the microtubule-organizing center within cloned natural killer cells bound to their targets. Proc Natl Acad Sci USA 80:7224-7228.
Lane JD, Lucocq J, Pryde J, Barr FA, Woodman PG, Allan VJ, Lowe M (2002) Caspase-mediated cleavage of the stacking protein GRASP65 is required for Golgi fragmentation during apoptosis. J Cell Biol 156:495-509.

Lecuit T, Pilot F (2003) Developmental control of cell morphogenesis: a focus on membrane growth. Nat Cell Biol 5:103-108.

Lefrancois L, Lyles DS (1982) The interaction of antibody with the major surface glycoprotein of vesicular stomatitis virus. Virology 121:157-167.

Li Z, Aizenman CD, Cline HT (2002) Regulation of rho GTPases by crosstalk and neuronal activity in vivo. Neuron 33:741-750.

Lippincott-Schwartz J, Roberts TH, Hirschberg K (2000) Secretory protein trafficking and organelle dynamics in living cells. Annu Rev Cell Dev Biol 16:557-589.

Maletic-Savatic M, Malinow R (1998) Calcium-evoked dendritic exocytosis in cultured hippocampal neurons. Part I: trans-Golgi network-derived organelles undergo regulated exocytosis. J Neurosci 18:6803-6813.

Martinez-Arca S, Coco S, Mainguy G, Schenk U, Alberts P, Bouille P, Mezzina M, Prochiantz A, Matteoli M, Louvard D, Galli T (2001) A common exocytotic mechanism mediates axonal and dendritic outgrowth. J Neurosci 21:3830-3838.

Matlin KS, Simons K (1983) Reduced temperature prevents transfer of a membrane glycoprotein to the cell surface but does not prevent terminal glycosylation. Cell 34:233-243.

Nakamura N, Rabouille C, Watson R, Nilsson T, Hui N, Slusarewicz P, Kreis TE, Warren G (1995) Characterization of a cis-Golgi matrix protein, GM130. J Cell Biol 131:1715-1726.

Nelson WJ, Yeaman C (2001) Protein trafficking in the exocytic pathway of polarized epithelial cells. Trends Cell Biol 11:483-486.

Nobes CD, Hall A (1999) Rho GTPases control polarity, protrusion, and adhesion during cell movement. J Cell Biol 144:1235-1244.

Passafaro M, Piech V, Sheng M (2001) Subunit-specific temporal and spatial patterns of AMPA receptor exocytosis in hippocampal neurons. Nat Neurosci 4:917-926.

Pelletier L, Jokitalo E, Warren G (2000) The effect of Golgi depletion on exocytic transport. Nat Cell Biol 2:840-846.

Pierce JP, van Leyen K, McCarthy JB (2000) Translocation machinery for synthesis of integral membrane and secretory proteins in dendritic spines. Nat Neurosci 3:311-313.

Pierce JP, Mayer T, McCarthy JB (2001) Evidence for a satellite secretory pathway in neuronal dendritic spines. Curr Biol 11:351-355.

Presley JF, Cole NB, Schroer TA, Hirschberg K, Zaal KJ, Lippincott-Schwartz J (1997) ER-to-Golgi transport visualized in living cells. Nature 389:81-85.

Rambourg A, Clermont Y (1990) Three-dimensional electron microscopy: structure of the Golgi apparatus. Eur J Cell Biol 51:189-200.

Rosenberg M, Meier J, Triller A, Vannier C (2001) Dynamics of glycine receptor insertion in the neuronal plasma membrane. J Neurosci 21:5036-5044.

Rossanese OW, Soderholm J, Bevis BJ, Sears IB, O’Connor J, Williamson EK, Glick BS (1999) Golgi structure correlates with transitional endoplasmic reticulum organization in Pichia pastoris and Saccharomyces cerevisiae. J Cell Biol 145:69-81.

Sampo B, Kaech S, Kunz S, Banker G (2003) Two distinct mechanisms target membrane proteins to the axonal surface. Neuron 37:611-624.

Scott EK, Luo L (2001) How do dendrites take their shape? Nat Neurosci 4:359-365.

Sesso A, Fujiwara DT, Jaeger M, Jaeger R, Li TC, Monteiro MM, Correa H, Ferreira MA, Schumacher RI, Belisario J, Kachar B, Chen EJ (1999) Structural elements common to mitosis and apoptosis. Tissue Cell 31:357-371.

Shorter J, Warren G (2002) Golgi architecture and inheritance. Annu Rev Cell Dev Biol 18:379-420.

Song HJ, Poo MM (1999) Signal transduction underlying growth cone guidance by diffusible factors. Curr Opin Neurobiol 9:355-363.

Stephens DJ, Pepperkok R (2001) Illuminating the secretory pathway: when do we need vesicles? J Cell Sci 114:1053-1059.

Stephens DJ, Lin-Marq N, Pagano A, Pepperkok R, Paccaud JP (2000) COPI-coated ER-to-Golgi transport complexes segregate from COPII in close proximity to ER exit sites. J Cell Sci 113:2177-2185.

Steward O, Worley P (2002) Local synthesis of proteins at synaptic sites on dendrites: role in synaptic plasticity and memory consolidation? Neurobiol Learn Mem 78:508-527. 
Storrie B, Nilsson T (2002) The Golgi apparatus: balancing new with old. Traffic 3:521-529.

Sutterlin C, Lin CY, Feng Y, Ferris DK, Erikson RL, Malhotra V (2001) Polo-like kinase is required for the fragmentation of pericentriolar Golgi stacks during mitosis. Proc Natl Acad Sci USA 98:9128-9132.

Tang BL, Peter F, Krijnse-Locker J, Low SH, Griffiths G, Hong W (1997) The mammalian homolog of yeast Sec13p is enriched in the intermediate compartment and is essential for protein transport from the endoplasmic reticulum to the Golgi apparatus. Mol Cell Biol 17:256-266.

Toomre D, Steyer JA, Keller P, Almers W, Simons K (2000) Fusion of constitutive membrane traffic with the cell surface observed by evanescent wave microscopy. J Cell Biol 149:33-40.

Torre ER, Steward O (1996) Protein synthesis within dendrites: glycosyla- tion of newly synthesized proteins in dendrites of hippocampal neurons in culture. J Neurosci 16:5967-5978.

Vaillant AR, Zanassi P, Walsh GS, Aumont A, Alonso A, Miller FD (2002) Signaling mechanisms underlying reversible, activity-dependent dendrite formation. Neuron 34:985-998.

Wu GY, Deisseroth K, Tsien RW (2001) Spaced stimuli stabilize MAPK pathway activation and its effects on dendritic morphology. Nat Neurosci 4:151-158.

Yacoubian TA, Lo DC (2000) Truncated and full-length TrkB receptors regulate distinct modes of dendritic growth. Nat Neurosci 3:342-349.

Zakharenko S, Popov S (1998) Dynamics of axonal microtubules regulate the topology of new membrane insertion into the growing neurites. J Cell Biol 143:1077-1086. 